\title{
The Higgs mode in disordered superconductors close to a quantum phase transition
}

\author{
Daniel Sherman ${ }^{1,2}{ }^{\dagger}$, Uwe S. Pracht ${ }^{2}$, Boris Gorshunov ${ }^{2,3,4}$, Shachaf Poran', John Jesudasan ${ }^{5}$, \\ Madhavi Chand ${ }^{5}$, Pratap Raychaudhuri ${ }^{5}$, Mason Swanson ${ }^{6}$, Nandini Trivedi ${ }^{6}$, Assa Auerbach ${ }^{7}$, \\ Marc Scheffler ${ }^{2}$, Aviad Frydman $^{1 \star}$ and Martin Dressel ${ }^{2}$
}

The concept of mass generation by means of the Higgs mechanism was strongly inspired by earlier works on the Meissner-Ochsenfeld effect in superconductors. In quantum field theory, the excitations of longitudinal components of the Higgs field manifest as massive Higgs bosons. The analogous Higgs mode in superconductors has not yet been observed owing to its rapid decay into particle-hole pairs. According to recent theories, however, the Higgs mode should decrease below the superconducting pairing gap $2 \Delta$ and become visible in two-dimensional systems close to the superconductorinsulator transition. For experimental verification, we measured the complex terahertz transmission and tunnelling density of states of various thin films of superconducting $\mathrm{NbN}$ and InO close to criticality. Comparing both techniques reveals a growing discrepancy between the finite $\mathbf{2} \Delta$ and the threshold energy for electromagnetic absorption, which vanishes critically towards the superconductor-insulator transition. We identify the excess absorption below $2 \Delta$ as strong evidence of the Higgs mode in two-dimensional quantum critical superconductors.

T he Higgs mechanism, which has great implications for recent developments in particle physics ${ }^{1}$, originates in Anderson's pioneering work on symmetry breaking with gauge fields in superconductors ${ }^{2}$. A superconductor spontaneously breaks continuous $\mathrm{U}(1)$ symmetry and acquires the well-known Mexican hat potential with a degenerate circle of minima described by the order parameter $\Psi=A \mathrm{e}^{i \varphi}$ (see Fig. 1a). Excitations from the ground state can be classified as transverse Nambu-Goldstone (phase) modes and massive longitudinal Higgs (amplitude) modes (see blue and red lines in Fig. 1a). In particle physics, the latter manifest themselves as the Higgs boson, which was recently discovered at CERN (ref. 3). Indications of a Higgs mode in correlated manybody systems have been found in one-dimensional charge-densitywave systems $s^{4}$, quantum antiferromagnets ${ }^{5}$ and two-dimensional superfluid to Mott transitions in cold atoms $s^{6}$. An amplitude mode, also named the Higgs mode, was theoretically predicted for superconductors ${ }^{7}$ and recently reported to be measured by pump-probe spectroscopy ${ }^{8}$. This amplitude mode describes pairing fluctuations, which are qualitatively distinct from the purely bosonic mode expected from the $\mathrm{O}(2)$ field theory. The Higgs-amplitude mode analogous to the high-energy Higgs boson has not yet been observed in superconductors. A partial reason is that in homogeneous, Bardeen-Cooper-Schrieffer (BCS) superconductors the Higgs mode is short-lived and decays to particle-hole (Bogoliubov) pairs ${ }^{9,10}$. Nevertheless, collective modes were recently predicted to be significant in strongly disordered superconductors ${ }^{11}$ and, in particular, it was shown ${ }^{12-14}$ that the Higgs mode softens but remains sufficiently sharp near a quantum critical point (QCP) in two dimensions as it is found to be a critical energy scale of the quantum phase transition. Hence, the Higgs mass can be reduced below twice the pairing gap, $2 \Delta$, making this mode experimentally visible. Such a critical point has been suggested to be relevant for the superconductor-insulator transition (SIT) in twodimensional films.

The desired quantum phase transition (QPT) from a superconductor to an insulator can be tuned by introducing disorder on atomic length scales. It has been shown both experimentally ${ }^{15-19}$ and theoretically ${ }^{20-22}$ that, although being morphologically homogeneous, with increasing disorder superconducting films can progressively become electronically granular on length scales comparable to the superconducting coherence length. Whereas for modest disorder the superconducting state is hardly affected, strong disorder near the QCP decomposes the homogeneous state into individual superconducting islands. In this scenario, the QPT takes place at the critical disorder when phase fluctuations between different islands destroy the global phase coherence and the superfluid density $\rho_{\mathrm{s}}$ vanishes on a macroscopic length scale $^{23}$. Consequently, the loss of global phase coherence does not necessarily cause the pairing gap $\Delta$ to close, as the decoupled islands still remain superconducting. The value of the critical temperature $T_{c}$ in the vicinity of the QPT is thus not defined by the opening of a gap in the quasiparticle density of states, but rather by the presence of a global phase coherence. Indeed, finite values of $\Delta$ in strongly disordered thin films were experimentally observed in tunnelling spectroscopy experiments where $T_{\mathrm{c}}$ was already vanishingly small on the superconducting side or even zero on the insulating side of the QPT (refs 18,24). Near the QPT one expects two critical energy scales: on the insulating side, a charge gap $\omega_{\text {pair }}$, which is the energy required to insert a Cooper pair into the pair insulator ${ }^{23}$, and on the superconducting side, the Higgs (amplitude) mass gap. Both energy scales should vanish at the QPT.

\footnotetext{
${ }^{1}$ Department of Physics, Bar Ilan University, Ramat Gan 52900, Israel. ${ }^{2} 1$. Physikalisches Institut, Universität Stuttgart, Pfaffenwaldring 57, 70550 Stuttgart, Germany. ${ }^{3}$ Moscow Institute of Physics and Technology, 141700 Dolgoprudny, Moscow Region, Russia. ${ }^{4}$ Prokhorov Institute of General Physics, Russian Academy of Sciences, Vavilov Street 38, 119991 Moscow, Russia. ${ }^{5}$ Tata Institute of Fundamental Research, Homi Bhabha Road, Colaba, Mumbai 400005 , India. ${ }^{6}$ Department of Physics, The Ohio State University, Columbus, Ohio 43210, USA. ${ }^{7}$ Physics Department, Technion, 32000 Haifa, Israel. †Present address: Center for Quantum Devices, Niels Bohr Institute, University of Copenhagen, 2100 Copenhagen, Denmark. *e-mail: aviad.frydman@gmail.com
} 

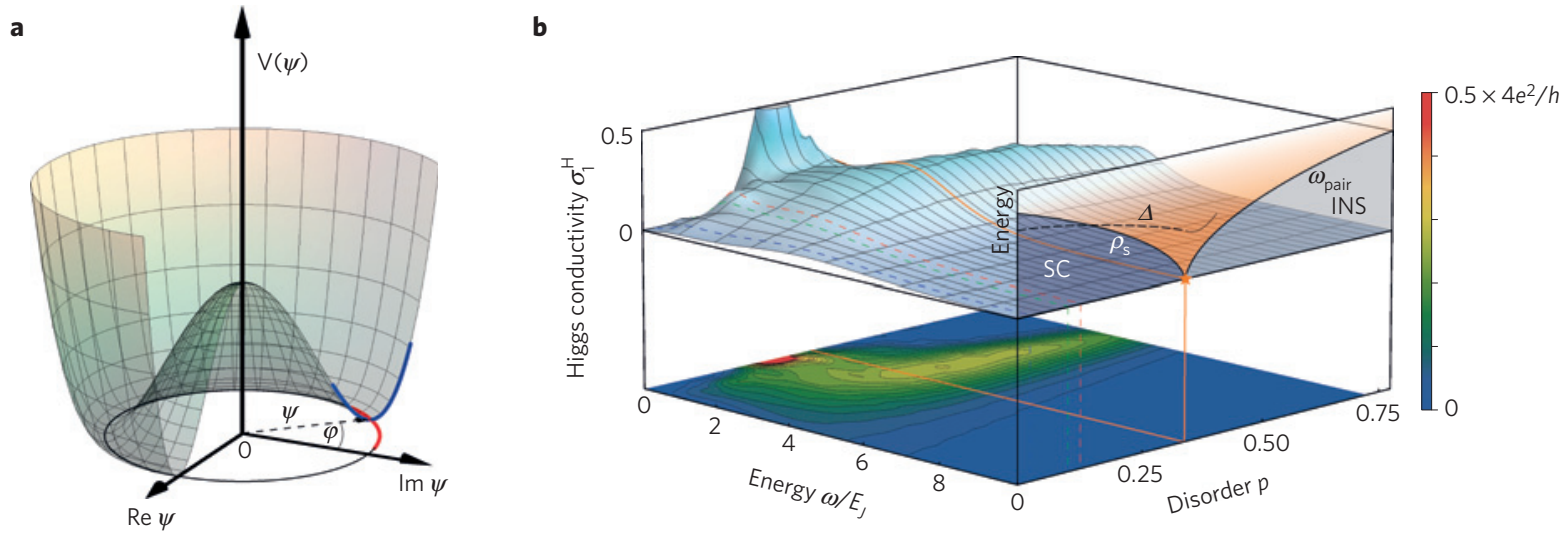

Figure 1 | Broken U(1)-symmetry phase and quantum Monte Carlo calculation of the Higgs conductivity. a, When symmetry is broken, the potential acquires a Mexican hat shape, with a circle of potential minima along the brim (black solid circle). Transverse modes of the order parameter $\Psi=A e^{i \varphi}$ along the brim (red line) are Nambu-Goldstone (phase) modes, and longitudinal modes (blue line) are Higgs (amplitude) modes associated with a finite energy. In superconductivity, the potential corresponds to the free energy. $\mathbf{b}$. The Higgs mode gives rise to low-frequency conductivity (in units of $4 e^{2} / h$ ) that grows as disorder $p$ (fraction of disconnected superconducting islands) is increased and remains finite through the quantum phase transition (orange line). At the quantum critical point, $p_{\mathrm{c}}=0.337$, the superfluid density, $\rho_{\mathrm{s}}$, in the superconducting phase vanishes and the quasiparticle gap, $\Delta$, remains finite, whereas in the insulator $\omega_{\text {pair, }}$ which is the energy to insert a Cooper pair to the insulator, goes to zero. Results for specific disorder (blue, green and red dashed lines) are compared to experiment (see Fig. 3). For details of the calculation see ref. 25.

Assuming the presence of a Higgs mode in the superconducting thin film, what would be the most suited experimental quantity to detect it? The Higgs mode is a finite-energy oscillation of the order parameter magnitude $|\Psi|$. It can be probed by the dynamical conductivity $\hat{\sigma}(\omega)$, which depends on the current-current correlation function $\langle[j(t), j(0)]\rangle$. At low temperatures, the current is dominated by the Cooper pair current $j \sim(2 e) \operatorname{Im}\left\{\Psi^{*} \nabla \Psi\right\} \simeq(2 e)|\Psi|^{2} \nabla \varphi$, where $\varphi$ is the local phase field and $e$ the elementary charge. As a result, the conductivity depends on a convolution of the amplitude and phase fluctuations.

How would the Higgs mode contribute to the dynamical conductivity? Theoretically it is predicted to give rise to excess conductivity at sub-gap frequencies ${ }^{12}$, which we will refer to as Higgs conductivity, $\hat{\sigma}^{\mathrm{H}}(\omega)$, in the remainder of the paper. In nondisordered systems ${ }^{13} \sigma_{1}^{\mathrm{H}}(\omega)$ shows a hard gap at frequencies similar to the superconducting gap, $\omega \sim 2 \Delta / \hbar$, that is associated with the energy scale of the Higgs mode, $m_{\mathrm{H}}$. This gap becomes softer as the system approaches the QPT, reaching zero at the critical point. Recently, Swanson and collaborators ${ }^{25}$ studied the effect of disorder on the dynamical conductivity across the superconductor-insulator QPT, employing quantum Monte Carlo methods, and extracted the excess low-frequency contribution (see Fig. 1b). The calculations show that the presence of disorder suppresses $m_{\mathrm{H}}$ such that $\sigma_{1}^{\mathrm{H}}(\omega)$ remains finite across the QPT. This excess conductivity adds to the conductivity stemming from the superfluid condensate and the quasiparticle dynamics, so that one can write

$$
\hat{\sigma}(\omega)=\sigma_{1}(\omega)+i \sigma_{2}(\omega)=\underbrace{A \rho_{s} \delta(\omega)+\hat{\sigma}^{q p}(\omega)}_{\hat{\sigma}^{B C S}(\omega)}+\hat{\sigma}^{\mathrm{H}}(\omega)
$$

where $\rho_{\mathrm{s}}$ is the superfluid density and $A$ is a constant ${ }^{26}$.

To experimentally search for the contribution of the Higgs mode, we have studied disordered superconducting films of $\mathrm{NbN}$ and InO by means of $\mathrm{THz}$ spectroscopy. Since the superconducting energy gaps are of the order of $0.1-1 \mathrm{THz}$, optical spectroscopy in this regime is an alternative method to tunnelling spectroscopy for the measurement of $2 \Delta$. Most importantly, unlike tunnelling, which measures the density of states of the quasiparticles, optical spectroscopy probes a complex response function, $\hat{\sigma}^{\exp }$, that combines those from the superfluid condensate, the quasiparticle dynamics and collective modes, see equation (1). One can decompose the optically measured conductivity into the regular BCS contribution and the contribution of the collective excitations. The first contribution is modelled by the Mattis-Bardeen theory for ordinary superconductors using our tunnelling spectroscopy results as input to fix the absolute numbers. The difference from the experimental data determines the Higgs mode, simply by calculating

$$
\sigma_{1}^{\mathrm{H}}(\omega)=\sigma_{1}^{\exp }(\omega)-\sigma_{1}^{\mathrm{BCS}}(\omega)
$$

We have measured the complex transmission coefficient of several thin-film samples with different degrees of disorder using Mach-Zehnder interferometry. Measurements were performed in the frequency domain between 0.05 and $1.2 \mathrm{THz}$ (corresponding to $1.7-40 \mathrm{~cm}^{-1}$ or $0.18-5 \mathrm{meV}$ ) for temperatures above and well below $T_{\mathrm{c}}$. From this we directly obtain the real and imaginary parts, $\sigma_{1}^{\exp }$ and $\sigma_{2}^{\text {exp }}$, of the dynamical conductivity, in a individual manner without Kramers-Kronig analysis. According to Mattis-Bardeen theory, $\sigma_{1}$ is minimal at a frequency $\Omega$ that corresponds to twice the superconducting energy gap, $2 \Delta$. Furthermore, the superfluid density is related to $\sigma_{2}(\omega)$ as

$$
\rho_{\mathrm{s}}=\frac{\sigma_{2}(\omega) m \omega}{e^{2}}
$$

where $m$ is the electron mass and $e$ is the elementary charge. This robust approach is well established to study superconducting thin films. For more details see Methods and, for example, refs 26-29. Figure $2 \mathrm{~b}$,e shows the real part of the conductivity $\sigma_{1}^{\exp }(\omega)$ for modestly $\left(T_{\mathrm{c}}=9.5 \mathrm{~K}\right)$ and strongly $\left(T_{\mathrm{c}}=4.2 \mathrm{~K}\right)$ disordered $\mathrm{NbN}$ in the normal state and well below $T_{c}$, together with the fits to the Mattis-Bardeen prediction for the disordered regime ${ }^{30}$. In both cases, $\sigma_{1}^{\exp }(\omega)$ is featureless in the normal state, following a simple Drude behaviour with a scattering rate well above the THz range, whereas $\sigma_{1}^{\exp }(\omega)$ is strongly suppressed in the superconducting state. The ordered sample is fitted perfectly by the Mattis-Bardeen theory. The onset of the high-frequency upturn coincides with twice the energy gap, $\Delta_{\mathrm{t}}$, obtained by tunnelling spectroscopy performed on a similar sample ${ }^{24}$, as seen in Fig. 2a. The situation is remarkably different for the strongly disordered sample. Here the decrease 


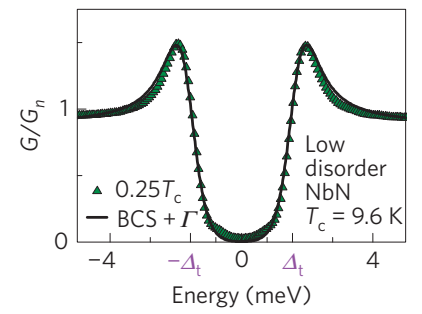

b

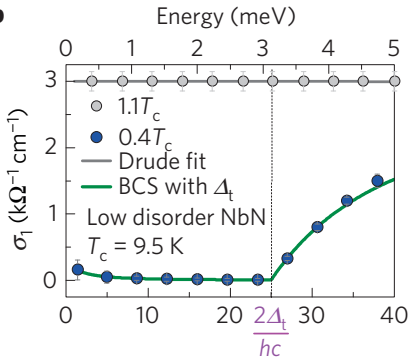

Frequency $\left(\mathrm{cm}^{-1}\right)$ c

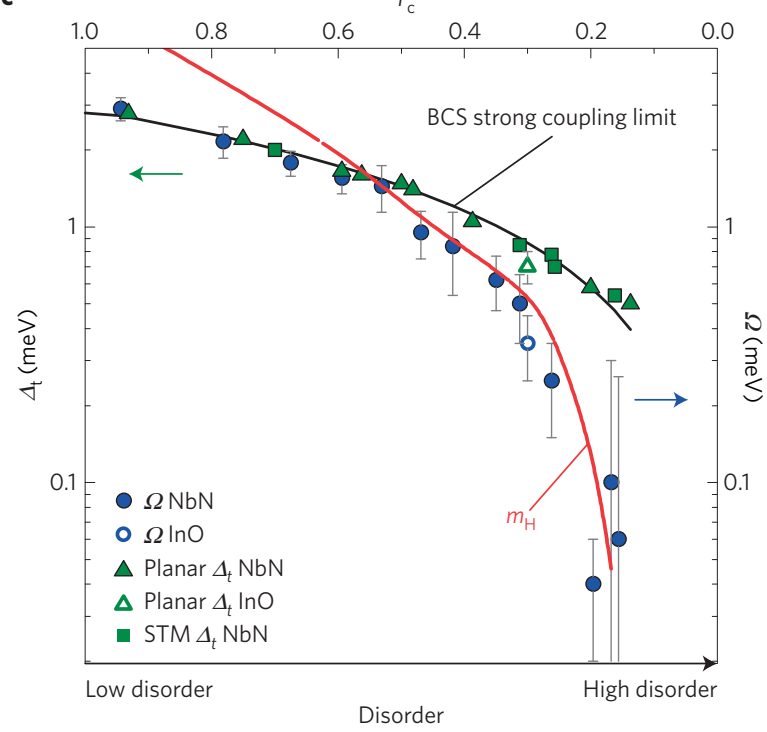

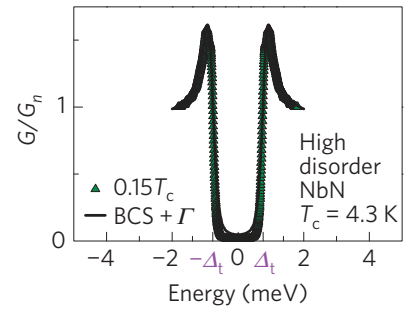

e

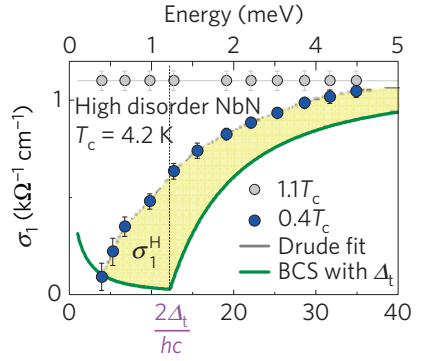

Frequency $\left(\mathrm{cm}^{-1}\right)$

Figure 2 | Tunnelling versus optical spectroscopy. a,b, Experimental results on low-disorder NbN samples. $\mathbf{a}$, Measured tunnelling conductance normalized to the normal state conductance $G / G_{n}$ (green triangles) alongside a fit to BCS (black line) with a Dynes broadening parameter, $\Gamma$. b, Real part of the dynamical conductivity, $\sigma_{1}$, versus frequency (energy) at temperatures below and above $T_{C}=9.5 \mathrm{~K}$. The low-temperature curve is fitted (green line) to Mattis-Bardeen theory using the energy gap value obtained in the corresponding tunnelling result, $\Delta_{\mathrm{t}}$. c, Summary of the quasiparticle tunnelling gap, $\Delta_{\mathrm{t}}$ (green symbols), measured by planar tunnelling junctions or scanning tunnelling microscopy (STM), versus $\Omega$, the frequency at which $\sigma_{1}(\omega)$ is minimal (blue symbols), obtained from optical spectroscopy for several superconducting NbN and InO films spanning the different degrees of disorder. Whereas the quasiparticle gap, $\Delta_{\mathrm{t}}$, remains fairly unchanged with increasing disorder, and basically falls on the BCS strong coupling limit ratio, $\Omega$ is significantly suppressed. According to Mattis-Bardeen theory, for ideal superconductors $\sigma_{1}$ is minimal at a frequency $\Omega$ that corresponds to $2 \Delta$. The discrepancy between both spectroscopic probes increases towards the highly disordered limit, signalling the presence of additional modes superimposed on the quasiparticle response. The solid red line corresponds to the analytical prediction of $m_{H}$ close to a QPT calculated by Podolsky and colleagues ${ }^{12}$. d,e, Experimental results on highly disordered $\mathrm{NbN}$ samples. d, Measured tunnelling conductance normalized to the normal state conductance $G / G_{n}$ (green triangles) together with a fit to BCS (black line) with a Dynes broadening parameter, $\Gamma$. e, Real part of the dynamical conductivity, $\sigma_{1}$, versus frequency (energy) at temperatures below and above $T_{\mathrm{c}}=4.2 \mathrm{~K}$. The low-temperature curve is fitted (green line) to Mattis-Bardeen theory using the energy gap value obtained in the corresponding tunnelling result. Unlike the case of the low-disorder sample, these two curves differ. The excess spectral weight, marked in yellow and defined as the difference between the curves, is attributed to the Higgs contribution, $\sigma_{1}^{\mathrm{H}}$ (see text). The error bars for $\sigma_{1}$ in the graphs are determined by the distortion of the Fabry-Perot oscillations due to parasitic radiation, standing waves and electronic noise.

towards low frequencies is not at all captured by BCS theory (green curve). In fact, using $\Delta_{\mathrm{t}}$ extracted from corresponding tunnelling experiments, as seen in Fig. 2d, yields a curve which is significantly below $\sigma_{1}^{\exp }(\omega)$. With increasing disorder, both the discrepancy between $2 \Delta_{\mathrm{t}}$ and $\Omega$ and the insufficiency of Mattis-Bardeen fits become progressively worse. This trend is demonstrated in Fig. 2c, where we compare results from both techniques on a large number of $\mathrm{NbN}$ and $\mathrm{InO}$ samples spanning the various degrees of disorder (measured in terms of the normalized critical temperature, $\left.\tilde{T}_{c}=T_{c} / T_{c}^{\text {clean }}\right)$. For small disorder, $\tilde{T}_{c} \simeq 1$, tunnelling and $\mathrm{THz}$ spectroscopy yield the same value for the superconducting energy gap. On increasing disorder (decreasing $\tilde{T}_{c}$ ) the discrepancy becomes more and more pronounced. For the most-disordered samples, we find about one order of magnitude difference between corresponding values. We assign these differences to an absorption process stemming from the Higgs mode that becomes progressively prominent as the system approaches the quantum critical point. This explains the discrepancy in the sense that $\Omega$ in the strong-disorder limit no longer equals $2 \Delta$ as a consequence of the additional conductivity $\sigma_{1}^{\mathrm{H}}(\omega)$ of the emergent Higgs mode. The previously prominent spectral feature marking the gap frequency is now hidden in the shoulder at higher frequencies. Although a distinct experimental determination of $\Omega$ becomes progressively difficult as it is pushed to low frequencies, we note the resemblance between $\Omega$ and the theoretical prediction of $m_{\mathrm{H}}$ in the vicinity of the critical point ${ }^{12}$, as seen in Fig. 2c.
We now explore the evolution of the observed additional excess weight associated with the Higgs conductivity, $\sigma_{1}^{\mathrm{H}}(\omega)$, as defined in equation (2), and compare these measured results with recent numerical simulations detailed in ref. 25 and sketched in Fig. $1 \mathrm{~b}$. Figure $3 \mathrm{a}$ shows the measured $\sigma_{1}^{\mathrm{H}}(\omega)$ for three disordered $\mathrm{NbN}$ films with different critical temperatures $T_{\mathrm{c}}=6.7,5$ and $4.2 \mathrm{~K}$ and the theoretical calculation for corresponding values of disorder $p=0.075,0.1$ and 0.125 . We note that one cannot expect a perfect quantitative agreement since the theory assumes that $2 \Delta$ is much larger than the Higgs mode energy, whereas experimentally they are of the same order of magnitude. Nevertheless, the overall behaviour-and even quantitative trends-is shared by theory and experiment: There is a pronounced peak of $\sigma_{\mathrm{H}}(\omega)$, which shifts towards smaller frequencies and becomes sharper with increasing disorder.

The appearance of the Higgs mode must go along with a redistribution of the spectral weight, as this quantity is strictly conserved; it measures the total charge carrier density $N$ in the system $^{26}$. In accordance with the bosonic model of the SIT sketched above, the strength of the $\delta$-peak-that is, the superfluid density $\rho_{\mathrm{s}}$-dwindles to zero in the vicinity of the quantum critical point. Figure $3 \mathrm{~b}$ shows $\rho_{\mathrm{s}}$ for disordered $\mathrm{NbN}$ films extracted from the imaginary part of the conductivity, using equation (3), and $N$ in the normal state obtained from Hall measurements. While $\rho_{\mathrm{s}}$ is reduced by about two orders or magnitude with increasing disorder, $N$ is much less affected. According to the Ferrell-Tinkham-Glover 
a

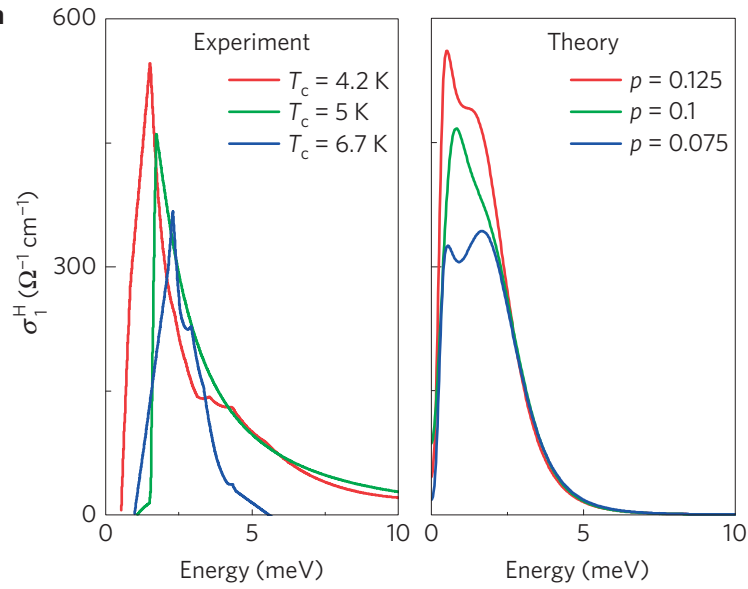

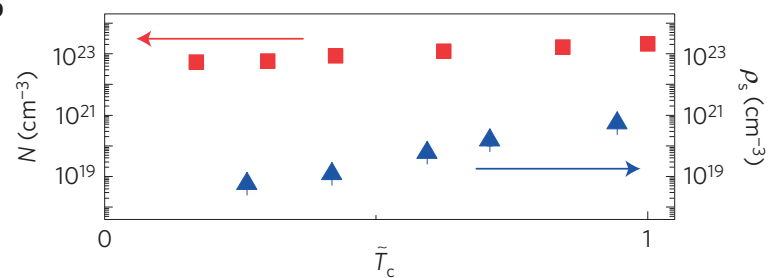

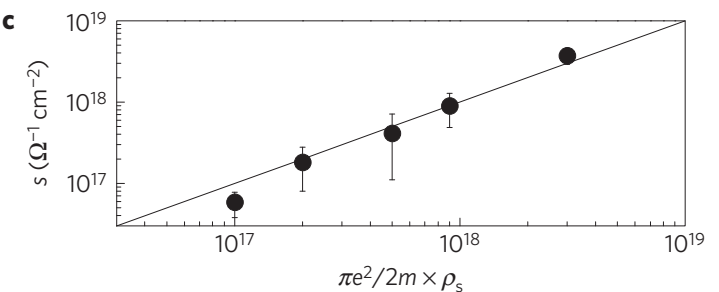

Figure 3 | Higgs conductivity and spectral weight. a, Experimental and theoretical results for the Higgs conductivity $\sigma_{1}^{H}$, as a function of energy for three $\mathrm{NbN}$ films of different disorder. The numerical results ${ }^{25}$ were obtained for a fixed value of $E_{C} / E_{J}$, whereas the degree of disorder, reflecting breaking bonds between the superconducting islands, is denoted by $p$. Qualitative and quantitative features are shared by both experiment and theory. The sharp lines in the experimental data are due to interpolation between measured data points. b. Charge carrier density $N$ in the normal state obtained from Hall measurements (red squares) and superfluid density, $\rho_{\mathrm{s}}$, measured by optical spectroscopy as functions of $T_{\mathrm{c}} / T_{\mathrm{c}}^{\text {clean }}$ (reflecting the degree of disorder). Note the faster decrease of $\rho_{\mathrm{s}}$ with increasing disorder, indicating the vanishing contribution of the superfluid condensate to the spectral weight. c, Redistribution of the 'missing' spectral weight $s$ between the normal and superconducting states versus the superfluid density $\rho_{\mathrm{s}}$, as defined in equation (4). The observed linear relation indicates that the redistribution of the spectral weight occurs within our measured energy spectrum.

sum rule ${ }^{26}$ for the 'missing' spectral weight $s$ between normal and superconducting states,

$$
s=\int_{0^{+}}^{\infty} \mathrm{d} \omega\left[\sigma_{1}^{n}(\omega)-\sigma_{1}^{s}(\omega)\right] \sim \rho_{\mathrm{s}}
$$

a reduced superfluid density $\rho_{\mathrm{s}}$ on increasing disorder leads to a reduced value of $s$. As the quasiparticle gap remains fairly unchanged with disorder, this necessarily causes the spectral weight contribution of the Higgs mode, $\int \mathrm{d} \omega \sigma_{1}^{\mathrm{H}}(\omega)$, to become more pronounced. Figure $3 c$ shows the detected linear relationship between the missing spectral weight, $s$, and the superfluid density, $\rho_{\mathrm{s}}$, for several films with different degrees of disorder, thus providing the self consistency of the above argument and eliminating the possibility of a redistributed spectral weight to higher frequencies (due to a sudden change in the scattering rate, for example).

We conclude that the low-frequency absorption observed by optical spectroscopy originates from the Higgs mode in superconductors close to a quantum phase transition. As the system approaches the critical point, the energy scale for this mode decreases and its magnitude grows, exhibiting quantitative agreement with numerical simulations.

The study of the properties of disordered superconductors is a subject of ongoing intense activity, mostly because it is viewed as being one of the few physical systems that can be tuned through a two-dimensional quantum critical point, which is not mean-field-like. The softening of the Higgs mode is direct proof that the SIT transition is a quantum critical point in which a diverging timescale is detected. Evidently, the vicinity to the QPT offers a unique opportunity to study the nature of the low-energy collective excitations in superconductors. Going beyond disordered superconductors, our findings can play a role in tracing collective excitations in other quantum critical condensed matter systems and might influence related fields such as Bose-condensed ultracold atoms, quantum statistical mechanics and high-energy physics.

\section{Methods}

The InO films were deposited on $10 \times 10 \mathrm{~mm}^{2}$ of $\mathrm{THz}$-transparent $\mathrm{MgO}$ or sapphire substrates (with various thickness ranging from 0.5 to $1.5 \mathrm{~mm}$ ) by e-gun evaporation. During the deposition process dry oxygen was injected into the chamber; the partial oxygen pressure allows us to tune the disorder. The $\mathrm{NbN}$ films were grown on similar $\mathrm{MgO}$ substrates by reactive magnetron sputtering, where the $\mathrm{Nb} / \mathrm{N}$ ratio in the plasma served as a disorder tuning parameter. In both cases the deposited films were structurally homogeneous; the thickness ranges from 15 to $40 \mathrm{~nm}$. DC transport measurements were used to characterize $T_{\mathrm{c}}$. THz spectroscopy has been applied in the past to confirm the BCS theory, as it probes the energy range of the superconducting gap ${ }^{27-29}$. The experimental set-up ${ }^{27,28}$ is based on several backward wave oscillators as powerful radiation sources to emit continuous-wave, coherent radiation which, in sum, can be tuned over the frequency range $0.05-1.2 \mathrm{THz}$, corresponding to a photon energy of $0.18-5 \mathrm{meV}$. We employ a quasi-optical Mach-Zehnder interferometer to measure the complex transmission $T=t \mathrm{e}^{i \theta}$, with $t$ the amplitude and $\theta$ the phase shift of radiation passing through the sample under study, from which the complex conductivity, $\hat{\sigma}(\omega)$, is directly calculated. The samples were mounted in an optical ${ }^{4} \mathrm{He}$ cryostat with a continuously accessible temperature range spanning from 300 to $1.85 \mathrm{~K}$. To further proceed with the experimental data, we employ two analysis routines. In the first, $t$ and $\theta$ are simultaneously fitted to a combination of Fresnel equations (for multiple reflections) ${ }^{26}$ for the optics and an appropriate microscopic model for the charge carrier dynamics (that is, Drude theory for the metallic state and BCS theory for the superconducting state, complemented by a finite scattering rate $^{30}$ ). Free-electron parameters (such as the scattering rate or plasma frequency) required for the BCS fit are taken from Drude fits to the normal-state $t$ and $\theta$ slightly above the superconducting transition. The superconducting energy gap $2 \Delta$ is then obtained as the sole fit parameter. Although this approach is well established for BCS-type-that is, non-disordered-superconducting systems, it fails for disordered systems beyond the Anderson limit. The second routine is suited for systems where no microscopic model is available-that is, strongly disordered systems. In a narrow band around each Fabry-Perot resonance (which are caused by the finite thickness of the sample), we fit $t$ and $\theta$ exclusively to the Fresnel equations using $\sigma_{1}$ and $\sigma_{2}$ as fit parameters. Depending on the optical thickness of the substrate this routine yields 10 to 15 pairs of $\sigma_{1}$ and $\sigma_{2}$ for each resonance frequency $\omega_{i}$. Details of the experimental set-up and analysis routines are found, for example, in refs $26-29,31$.

Received 7 July 2014; accepted 11 December 2014; published online 26 January 2015

\section{References}

1. Álvarez-Gaumé, L. \& Ellis, J. Eyes on a prize particle. Nature Phys. 7, 2-3 (2011).

2. Anderson, P. W. Plasmons, gauge invariance and mass. Phys. Rev. 130, 439-442 (1963). 
3. ATLAS Collaboration, Observation of a new particle in the search for the Standard Model Higgs boson with the ATLAS detector at the LHC. Phys. Lett. B 716, 1-29 (2012).

4. Sooryakumar, R. \& Klein, M. V. Raman scattering by superconducting-gap excitations and their coupling to charge-density waves. Phys. Rev. Lett. 45, 660-662 (1980)

5. Rüegg, Ch. et al. Quantum magnets under pressure: Controlling elementary excitations in $\mathrm{TlCuCl}_{3}$. Phys. Rev. Lett. 100, 205701 (2008).

6. Endres, M. et al. The 'Higgs' amplitude mode at the two-dimensional superfluid/Mott insulator transition. Nature 487, 454-458 (2012).

7. Littlewood, P. B. \& Varma, C. M. Amplitude collective modes in superconductors and their coupling to charge density waves. Phys. Rev. B. 26, 4883-4893 (1982).

8. Matsunaga, R. et al. Higgs amplitude mode in the BCS superconductors $\mathrm{Nb}_{1-x} \mathrm{Ti}_{x} \mathrm{~N}$ induced by terahertz pulse excitation. Phys. Rev. Lett. 111 057002 (2013).

9. Sachdev, S. Universal relaxational dynamics near two-dimensional quantum critical points. Phys. Rev. B 59, 14054-14073 (1999).

10. Zwerger, W. Anomalous fluctuations in phases with a broken continuous symmetry. Phys. Rev. Lett. 92, 027203 (2004).

11. Cea, T. et al. Optical excitation of phase modes in strongly disordered superconductors. Phys. Rev. B 89, 174506 (2014).

12. Podolsky, D., Auerbach, A. \& Arovas, D. P. Visibility of the amplitude (Higgs) mode in condensed matter. Phys. Rev. B 84, 174522 (2011).

13. Gazit, S., Podolsky, D., Auerbach, A. \& Arovas, D. P. Dynamics and conductivity near quantum criticality. Phys. Rev. B 88, 235108 (2013).

14. Gazit, S., Podolsky, D. \& Auerbach, A. Fate of the Higgs mode near quantum criticality. Phys. Rev. Lett. 110, 140401 (2013).

15. Kowal, D. \& Ovadyahu, Z. Disorder induced granularity in an amorphous superconductor. Solid State Commun. 90, 783-786 (1994).

16. Sacepe, B. et al. Disorder-induced inhomogeneities of the superconducting state close to the superconductor-insulator transition. Phys. Rev. Lett. 101, 157006 (2008).

17. Kamlapure, A. et al. Emergence of nanoscale inhomogeneity in the superconducting state of a homogeneously disordered conventional superconductor. Sci. Rep. 3, 2979 (2013).

18. Sherman, D., Kopnov, G., Shahar, D. \& Frydman, A. Measurement of a superconducting energy gap in a homogeneously amorphous insulator. Phys. Rev. Lett. 108, 177006 (2012).

19. Chand, M. et al. Phase diagram of the strongly disordered s-wave superconductor NbN close to the metal-insulator transition. Phys. Rev. B 85, 014508 (2012).

20. Ghosal, A., Randeria, M. \& Trivedi, N. Role of spatial amplitude fluctuations in highly disordered s-wave superconductors. Phys. Rev. Lett. 81, 3940-3943 (1998).

21. Ghosal, A., Randeria, M. \& Trivedi, N. Inhomogeneous pairing in highly disordered s-wave superconductors. Phys. Rev. B 65, 014501 (2001)

22. Dubi, Y., Meir, Y. \& Avishai, Y. Nature of the superconductor-insulator transition in disordered superconductors. Nature 449, 876-880 (2007).
23. Bouadim, K., Loh, Y., Randeria, M. \& Trivedi, N. Single and two-particle energy gaps across the disorder-driven superconductor-insulator transition. Nature Phys. 11, 884-889 (2011).

24. Mondal, M. et al. Phase fluctuations in a strongly disordered s-wave NbN superconductor close to the metal-insulator transition. Phys. Rev. Lett. 106, 047001 (2011)

25. Swanson, M., Loh, Y. L., Randeria, M. \& Trivedi, N. Dynamical conductivity across the disorder-tuned superconductor-insulator transition. Phys. Rev. X 4 021007 (2014).

26. Dressel, M. \& Grüner, G. Electrodynamics of Solids (Cambridge Univ. Press, 2002).

27. Pracht, U. S. et al. Electrodynamics of the superconducting state in ultra-thin films at THz frequencies. IEEE Trans. THz Sci. Technol. 3, 269-280 (2013).

28. Dressel, M., Drichko, N., Gorshunov, B. \& Pimenov, A. THz spectroscopy of superconductors. IEEE J. Sel. Topics Quantum Electron. 14, 399-406 (2008).

29. Pracht, U. S. et al. Direct observation of the superconducting gap in a thin film of titanium nitride using terahertz spectroscopy. Phys. Rev. B 86, 184503 (2012)

30. Zimmermann, W., Brandt, E. H., Bauer, M., Seider, E. \& Genzel, L. Optical conductivity of BCS superconductors with arbitrary purity. Physica C $\mathbf{1 8 3}$ 99-104 (1991).

31. Sherman, D. et al. Effect of Coulomb interactions on the disorder-driven superconductor-insulator transition. Phys. Rev. B 89, 035149 (2014).

\section{Acknowledgements}

We are grateful for useful discussions with D. Arovas, L. Benfatto, S. Gazit, D. Podolsky and E. Shimshoni. We acknowledge support from the the GIF foundation grant I-1250-303.10/2014 and from the Deutsche Forschungsgemeinschaft. U.S.P. acknowledges financial support from the Studienstiftung des deutschen Volkes. B.G. acknowledges support from the Russian Ministry of Education and Science (Program 5 top 100) and A.A. acknowledges support from the ISF and BSF foundations. M.S. acknowledges support from the NSF Graduate Research Fellowship and N.T. acknowledges support from grant DOE DE-FG02-07ER46423 (N.T.) and computational support from the Ohio Supercomputing Center.

\section{Author contributions}

D.S., J.J., M.C. and P.R. carried out the DC experiments. D.S., U.S.P. and B.G. carried out the THz experiments. D.S., U.S.P. and A.F. analysed the data. D.S., S.P., J.J. and P.R. prepared the samples. N.T., M.S. and A.A. carried out the theoretical analysis and the numerical simulations. U.S.P., D.S., M.D., A.F., M.S., N.T. and A.A. wrote the paper. A.F. and M.D. initiated and supervised the work. All the authors discussed the results and commented on the manuscript.

\section{Additional information}

Reprints and permissions information is available online at www.nature.com/reprints. Correspondence and requests for materials should be addressed to A.F.

\section{Competing financial interests}

The authors declare no competing financial interests. 